Como citar: Fiuza, W.M., \& de Godoy, R. F. (2021). Esquemas iniciais desadaptativos em adultos brasileiros: revisão narrativa da literatura. PSI UNISC, 5(2), 59-77. doi: 10.17058/psiunisc.v5i2.16416

\title{
Esquemas iniciais desadaptativos em adultos brasileiros: revisão narrativa da literatura
}

\author{
Esquemas tempranos desadaptativos en adultos brasileños: revisión de la literatura \\ narrativa
}

Early maladaptive schemas in Brazilian adults: narrative literature review

William Macedo Fiuza

Universidade de Caxias do Sul (UCS), Caxias do Sul - RS/Brasil ORCID: 0000-0002-7688-7192

E-mail: fiuzawm@gmail.com

Rossane Frizzo de Godoy
Universidade de Caxias do Sul (UCS), Caxias do Sul - RS/Brasil
ORCID: 0000-0002-6753-4280
E-mail: rfgodoy@ucs.br

\section{Resumo}

O objetivo deste artigo foi identificar estudos produzidos no Brasil que tenham investigado esquemas iniciais desadaptativos em adultos. O delineamento foi qualitativo-exploratório, por meio de uma revisão narrativa da literatura, tendo como fontes estudos empíricos produzidos no Brasil publicados no período de 2010 à 2020, nas seguintes bases de dados: PsycINFO, BVS-PSI e o portal de periódicos da CAPES. Foram selecionados 16 artigos que investigaram a relação dos EID's com os seguintes grupos: homens e mulheres, pais, mães e habilidades sociais educativas, universitários e empatia, sintomas depressivos, ansiosos e dismorfismo corporal em universitários, bullying em universitários, pacientes com enxaqueca, uso de álcool e outras drogas, transtorno da acumulação, violência conjugal, ajustamento diádico na conjugalidade, pacientes bariátricos, pessoas com sintomas de disfunção temporomandibular, pacientes internados por tentativa de suicídio e indivíduos que praticaram crimes. Uma das temáticas mais recorrentes foi a violência conjugal. Nos três estudos que abordaram o assunto, foi identificada uma possível associação com o $1^{\circ}$ domínio esquemático (desconexão e rejeição). Foi observado o aumento gradativo de estudos brasileiros com a identificação de EID's e o uso das versões reduzidas do inventário de esquemas de Young. Como limitação deste artigo, percebe-se a variedade de métodos nos estudos registrados, o que dificultou uma análise mais detalhada de tais produções. Ampliar esse campo de pesquisa pode favorecer entendimentos de diferentes contextos sob o viés da terapia do esquema, possibilitando intervenções terapêuticas e de prevenção mais efetivas.

Palavras-chaves: Esquemas iniciais desadaptativos; Terapia do esquema; População brasileira; Adultos.

\section{Resumen}

El objetivo de este artículo fue identificar los estudios realizados en Brasil que investigaron los esquemas tempranos inadaptados (ETI) en adultos. El diseño fue cualitativo-exploratorio, a través de una revisión narrativa de la literatura, utilizando como fuentes estudios empíricos producidos en Brasil publicados en el período de 2010 a 2020, con las siguientes bases de datos: PsycINFO, BVS-PSI y portal de revistas CAPES. Seleccionamos 16 artículos que investigarán la relación entre dos ETIs en los siguientes grupos: : hombres y mujeres, padres, madres y habilidades sociales educativas. , estudiantes universitarios y empatía, síntomas depresivos, ansiosos y dismorfismos corporales en estudiantes universitarios, bullying en estudiantes universitarios, pacientes con migraña, consumo de alcohol y otras drogas, trastorno de acumulación, violencia conyugal, ajuste diádico en conyugalidad, pacientes bariátricos, personas con síntomas de trastornos temporomandibulares, pacientes hospitalizados por intento de suicidio y personas que cometieron delitos. Algunos de los temas más recurrentes estaban relacionados con la violencia doméstica. En los tres estudios para abordar el problema se identificaron como una posible asociación con el primer dominio esquemático (desconexión y rechazo). Hay un aumento gradual de estudios brasileños con la identificación de EIT y el uso de versiones reducidas del 
inventario de esquemas de Young. Como limitación de este artículo, es posible notar la variedad de métodos en los estudios registrados, lo que dificultó un análisis más exhaustivo de tales producciones. La expansión de este campo de investigación puede favorecer la comprensión de diferentes contextos bajo el sesgo de la terapia de esquemas, permitiendo intervenciones terapéuticas y preventivas más efectivas.

Palabras clave: Esquemas tempranos inadaptados; Terapia de esquemas; Población brasileña; Adultos.

\begin{abstract}
The aim of this article was to identifiy studies produced in Brazil that have investigated maladaptive early schemas in adults. This was a qualitative-exploratory, through a narrative review of the liter: PsycINFO, BVSPSI and the CAPES journal portal. 16 artciles were selected and they were studying the ature, using empirical studies produced in Brazil between 2010 to 2020, using this databases relationship beetween EMS's and this groups: mans and womans, fathers, mothers and educational social skills, university students and
\end{abstract}

empathy, depressive symptoms, anxiety and corporal dismorphism in undergraduates, bullying in college students., patients with migraine, use of alcohol and other drugs, accumulation disorder, marital violence, dyadic adjustment in conjugality, bariatric patients, people with temporomandibular disorder symptoms, hospitalized patients for suicide attempt and criminal convicts. Marital violence was one of the subjects more used. In the three studies of this area a possible association with the $1^{\circ}$ schema domain (disconnection and rejection) was identify. Was identify a gradative growing in schema therapy studies in Brazil and the use of the Young Schema Questionnaire - short version. The variety of method used in the studies is a limitation of this article, making difficult to analyze them with more details. Expanding this kind of research may contribute to understand different contexts, making possible do effective preventive and therapeutic interventions using schema therapy approach.

Keywords: Early maladaptive schemas; Schema therapy; Brazilian population; Adults.

\section{Introdução}

A terapia do esquema (TE) é uma abordagem cognitivo-comportamental integrativa que visa utilizar preceitos da terapia cognitivo-comportamental tradicional, tais como processo terapêutico estruturado, com foco em solucionar problemas do presente, uso da psicoeducação para desenvolvimento da autonomia do paciente e o entendimento acerca da influência dos pensamentos no comportamento. Além disso, busca dar maior ênfase nas experiências da infância e na relação terapêutica como veículo de mudança, sendo as ativações emocionais ocorridas em terapia uma parte importante do processo. Para isso, a TE incorpora fundamentos de outras abordagens, como a Gestatl terapia, com o uso de imagens mentais e a conexão e contato com as as próprias emoções, e o entendimento psicanálitico acerca da tendência à repetição das conflitivas infantis na vida adulta (Young, Klosko \& Weishaar, 2008).

Uma das bases teóricas centrais que fundamenta a terapia do esquema é a teoria do apego, desenvolvida por John Bowlby, com contribuição de diversos autores, incluindo Mary Ainsworth. Em síntese, a teoria do apego propõe que a qualidade relacional vivenciada com os vínculos primários pode regular os comportamentos da criança e formar modelos internos de funcionamento que buscam mapear e compreender a dinâmica do mundo externo. O entendimento dessas relações de apego, compostas por segurança, estabilidade e afeto, além das consequências da falta destes elementos, tem papel fundamental na estruturação do que posteriormente chamou-se de esquemas inicias desadaptaitvos (EID's) e da relação terapêutica proposta na TE (Halperin \& Carneiro, 2016; Mendes \& Maia, 2019).

Um esquema, nesse contexto, é um padrão de funcionamento emocional composto por memórias, cognições e sensações corporais que acontecem de modo amplo e persistente na vida do sujeito. São nomeados como iniciais, ou remotos, por terem relação com experiências primárias do desenvolvimento e considerados desadaptativos pelos prejuízos significativos que geram para o indivíduo na vida adulta (Wainer, 2016; Young et al., 2008).

Múltiplos fatores contribuem para o desenvolvimento de um esquema. Embora o temperamento tenha uma forte influência nas necessidades emocionais da criança, é na 
relação com os cuidadores que o indivíduo aprende a se relacionar com o mundo e interioriza o quanto tais necessidades podem ou não ser atendidas (Young \& Klosko, 2019). Sendo assim, "experiências ambientais precoces extremamente favoráveis ou adversas podem alterar o temperamento emocional" (Falcone, 2014, p.271).

Ao longo do desenvolvimento infantil, existem tarefas evolutivas que precisam ser cumpridas, sendo elas: aceitação e pertencimento, senso de autonomia e competências, limites realistas, respeito aos seus desejos e aspirações e expressão emocional legítima. Quando o ambiente, especialmente na figura dos principais cuidadores, não consegue suprir essas necessidades emocionais de modos satisfatório, os esquemas iniciais desadaptativos podem surgir e se fortalecer por experiências significativas vivenciadas dentro e fora do ambiente familiar até o final da adolescência. Os esquemas podem ser incondicionais, mais remotos e nucleares, ou condicionais, desenvolvidos na tentativa de obter alívio ao sofrimento trazido pelos esquemas incondicionais (Young et al., 2008).

Na vida adulta, os EID's se mantêm e autoperpetuam, como se lutassem para sobreviver, por meio de três mecanismos básicos. O primeiro deles é expresso nas distorções cognitivas, que interpretam as informações do ambiente apenas de modo condizente com os esquemas. Já os padrões de vida autoderrotistas são comportamentos prejudiciais que ativam e reforçam os esquemas. Por fim, as estratégias de enfrentamento - hipercompensação, evitação e resignação - visam lidar com o esquema ativado, sendo as três principais estratégias correspondentes às respostas básicas do ser humano frente à ameaça: luta, fuga ou paralisação (Young et al., 2008). Apesar desses mecanismos serem úteis e protetivos na infância, ajudando a lidar com as condições adversas do ambiente, trazem prejuízos no contexto atual de vida, especialmente pela rigidez com que essas respostas acontecem (Fassbinder, Schweiger, Martius, Wilde \&
Arntz, 2016; Wainer, 2016).

Atualmente, compreende-se a existência de 18 esquemas iniciais desadaptativos (EID's), com características particulares, divididos em cinco domínios esquemáticos, conforme as necessidades emocionais não atendidas. A seguir são apresentados sinteticamente os cinco domínios esquemáticos e os EID's correspondentes, segundo o proposto por Young et al. (2008):

1. Desconexão e rejeição: família geralmente fria, rejeitadora, isoladora, imprevisível e/ou abusadora. Cuidadores que não conseguiram suprir a tarefa evolutiva de aceitação e pertencimento. Esse domínio é composto pelos seguintes EID's: abandono/instabilidade,

desconfiança/abuso, privação emocional, defectividade/vergonha, isolamento social/alienação.

2. Autonomia e desempenho prejudicados: família geralmente superprotetora, emaranhada e destruidora da confiança da criança. Cuidadores que não conseguiram suprir a tarefa evolutiva de senso de autonomia e competência adequados. Esse domínio é composto pelos seguintes EID's: fracasso, vulnerabilidade ao dano/doença,

dependência/incompetência e emaranhamento.

3. Limites prejudicados: família geralmente permissiva, faltando orientação à criança quanto ao que é certo e errado. Cuidadores que não conseguiram suprir a tarefa evolutiva de limites realistas. Esse domínio é composto pelos seguintes EID's: autocontrole/autodisciplina insuficientes grandiosidade/arrogo.

4. Orientação para o outro: família geralmente com relacionamentos 
baseados na aceitação condicional, anulando caracterísitcas e desejos próprios para obter amor ou aceitação social. Cuidadores que não conseguiram suprir a tarefa evolutiva de respeito aos próprios desejos e aspirações. Esse domínio é composto pelos seguintes EID's: subjugação, autossacrifício e busca de aprovação/reconhecimento.

5. Supervigilância e inibição: família geralmente punitiva, perfeccionista e tende a evitar "erros" decorrentes de atividades prazerosas, preocupando-se que as coisas darão errado se houver falha na vigilância. Cuidadores que não conseguiram suprir a tarefa evolutiva de expressão emocional legítima. Esse domínio é composto pelos seguintes EID's: inibição emocional, padrões inflexíveis/hipercriticidade,

negativismo/pessismismo e postura punitiva.

A identificação dos EID's é parte fundamental do processo da terapia do esquema. Para isso, é possível utilizar diversos recursos de avaliação, tais como: dados da história do indivíduo, trabalho com imagens mentais, análise situacional e automonitoramento, além da própria relação terapêutica (Farrell, Reis \& Shaw, 2014; Young et al., 2008). Inventários específicos acerca dos estilos parentais e dos EID's auxiliam na investigação quantitativa dos esquemas. Para adultos, além do inventário de esquemas de Young (Young Schema Questionnaire - YSQ) completo, existem versões reduzidas que permitem a investigação dos elementos mais representativos de cada esquema. Enquanto o YSQ-S2 avalia 15 EID's por meio de 75 questões, o YSQ-S3 avalia 18 EID's por intermédio de 90 questões (Cazassa \& Oliveira, 2008; Cazassa \& Oliveira, 2012; Paim \& Copetti, 2016).

De acordo com Taylor, Bee e Haddock (2015), apesar do foco inicial voltado para os transtornos de personalidade, a aplicação da terapia do esquema vem sendo desenvolvida e estudada em outros transtornos mentais, como a depressão crônica (Carter et al., 2013; Malogiannis et al., 2014; Renner et al., 2018), o transtorno bipolar (Hawke, Provencher \& Parikh, 2013), o transtorno do estresse póstraumático (Haan, Fassbinder, Hayes \& Lee, 2019), o transtorno obsessivo-compulsivo (Thiel et al., 2016) e os transtornos alimentares (Simpson, Morrow, Vreeswijk \& Reid, 2010; Pugh, 2015). Para que a TE estruture seu trabalho nas diferentes demandas clínicas, é necessário que possa compreender a relação que os EID's possuem com determinados quadros psicológicos (Masley, Gillanders, Simpson \& Taylor, 2012).

Isso ocorre pois conhecer possiveis padrões esquemáticos em populações específicas pode ser relevante no que diz respeito ao entendimento de determinado contexto e suas particularidades, bem como, suas diferenças com outras demandas. Portanto, isso também tem influência no planejamento de intervenções psicoterápicas específicas para cada público, tanto em contexto de psicoterapia quanto na formação de programas de atendimento e políticas públicas (Cazassa \& Oliveira, 2008; Luz, Santos, Cazassa \& Oliveira, 2012). Existem produções internacionais que possuem como objetivo investigar a prevalência de EID's em populações específicas. Alguns exemplos de demandas estudadas nesse sentido são: ansiedade social (Diez, Zumalde \& Sola, 2012), transtorno obsessivo-compulsivo e trânstorno do pânico (Kwak \& Lee, 2015) e sintomas psicóticos (Sundag, Ascone, Marques, Moritz \& Lincoln, 2016).

Atualmente, o entendimento de diferentes contextos por meio dos EID's também é utilizado para compreender fenômenos que não necessariamente estão ligados á patologias, como por exemplo, o relacionamento conjugal (Bohn, Berlitz, Oliveira \& Pureza, 2018) e a relação afetiva entre professores e alunos (Jager \& Macedo, 2018). De acordo com Videler, van Royen, Legra e Ouwens (2020), também estão sendo discutidos modelos teóricos que consideram a 
existência de esquemas adaptativos (positive schemas).

Porém, no Brasil, pesquisas com o objetivo de identificar EID's em diferentes sujeitos ainda são incipientes e cada cultura possui particularidades que devem ser consideradas (Luz et al., 2012). Desse modo, o objetivo do estudo foi identificar estudos produzidos no Brasil que tenham investigado esquemas iniciais desadaptativos em adultos.

\section{Método}

Foi realizado um estudo qualitativoexploratóro, por meio de uma revisão narrativa da literatura, a qual permite explorar diferentes perspectivas apresentadas em produções de determinado tema, propondo uma síntese das informações obtidas (Hohendorff, 2014).

As fontes utilizadas foram estudos empíricos produzidos no Brasil, publicados no período de 2010 à 2020, que tivessem como foco de pesquisa a identificação de esquemas iniciais desadaptativos em adultos. Foram utilizados os seguintes descritores com operadores booleanos: terapia do esquema, esquemas iniciais desadaptativos, esquemas desadaptativos remotos, esquemas iniciais desadaptativos em adultos. As bases de dados consultadas foram a PsycINFO, a Biblioteca Virtual em Saúde em Psicologia (BVS-PSI), que hospeda outras bases de dados como o Scielo e o Lilacs, e o portal de periódicos da Coordenação de Aperfeiçoamento de Pessoal de Nível Superior (CAPES), que hospeda o $P U B$-med. Foram incluídos no estudo artigos com relato de pesquisas empíricas brasileiras que identificassem os esquemas iniciais desadaptativos em populações adultas. Foram excluídos estudos teóricos, teses, dissertações, estudos com populações não brasileiras, estudos que abordassem outras fases do ciclo vital e estudos duplicados. Após a seleção dos artigos e leitura dos resumos, foram selecionados os estudos que atendiam aos critérios de inclusão e exclusão. Como instrumento para que as informações pudessem ser visualizadas, foi utilizada uma ficha de catalogação com as seguintes informações: autores e ano de publicação, natureza do estudo, objetivos, participantes, síntese dos resultados. Por fim, buscou-se integrar o conteúdo propondo-se uma síntese a partir dos resultados obtidos.

\section{Resultados}

Foram encontradas 23 publicações e, após avaliá-las a partir dos critérios de inclusão e exclusão, foram selecionados 16 artigos para análise, apresentados na Tabela 1. Destes, nove foram publicados em língua portuguesa e três em língua inglesa. Também foi possível observar o aumento gradativo de publicações no decorrer dos anos selecionados no período de busca. Nos primeiros seis anos (2010-2015) foram encontrados seis artigos e nos últimos cinco anos (2016-2020), foram encontrados dez artigos, sendo que 2018 é o ano que concentra o maior número de artigos (quatro) avaliados nessa revisão.

Os contextos investigados pelos artigos foram: homens e mulheres, pais, mães e habilidades sociais educativas, universitários e empatia, sintomas depressivos, ansiosos e dismorfismo corporal em universitários, bullying em universitários, pacientes com enxaqueca, uso de álcool e outras drogas, transtorno da acumulação, violência conjugal, ajustamento diádico, pacientes bariátricos, pessoas com sintomas de disfunção temporomandibular, pacientes internados por tentativa de suicídio e indivíduos que praticaram crimes.

Apesar das diferentes demandas apresentadas na Tabela 1, é possível observar semelhanças nos públicos investigados. Dois artigos apresentaram a comparação de esquemas entre homens e mulheres. Três estudos tiveram como foco o público universitário. Duas pesquisas foram realizadas com pessoas institucionalizadas e três abordavam a violência conjugal. 11 artigos abordaram questões relacionadas a situações de sofrimento, sintomatologia e quadros de transtorno mental.

O delineamento prevalente nos estudos 
foi o quantitativo, com amostras que variaram entre 16 e 372 participantes, havendo também um estudo misto (qualitativo-quantitativo) e um estudo de caso. Como instrumento para avaliação dos EID's foram utilizadas as versões breves do Inventário dos Esquemas de Young, com dez estudos optando pelo uso do YSQ-S2 sendo que um deles também fez uso do inventário de estilos parentais. Os seis estudos restantes utilizaram o modelo YSQ-S3.

Tabela 1

Pesqisas de identificação dos EID's em adultos

\begin{tabular}{|c|c|c|c|c|c|}
\hline Autores & $\begin{array}{l}\text { Natureza do } \\
\text { estudo }\end{array}$ & Objetivos & $\begin{array}{c}\text { Instumentos } \\
\text { utilizados } \\
\text { para } \\
\text { identificaçã } \\
\text { o dos EID's } \\
\end{array}$ & Participantes & Síntese dos Resultados \\
\hline $\begin{array}{l}\text { Barbosa, } \\
\text { Côrrea, } \\
\text { Zimmer e } \\
\text { Paludo } \\
\text { (2019) }\end{array}$ & $\begin{array}{c}\text { Qualitativa e } \\
\text { transversal }\end{array}$ & $\begin{array}{l}\text { Identificar domínios } \\
\text { esquemáticos e } \\
\text { tipos de violência } \\
\text { sofrida em um } \\
\text { grupo de } \\
\text { mulheres }\end{array}$ & YSQ-S3 & $\begin{array}{l}16 \text { mulheres } \\
\text { em situação de } \\
\text { violência } \\
\text { conjugal }\end{array}$ & $\begin{array}{l}\text { O domínio mais pontuado foi } \\
\text { o de desconexão e rejeição } \\
\text { (com os esquemas mais altos o } \\
\text { de abandono/insabilidade e } \\
\text { desconfiança/abuso), seguido } \\
\text { do domínio supervigilância e } \\
\text { inibição (com os esquemas } \\
\text { mais altos o de negativismo e } \\
\text { inibição emocional). }\end{array}$ \\
\hline $\begin{array}{l}\text { Berlitz e } \\
\text { Pureza } \\
(2018)\end{array}$ & $\begin{array}{l}\text { Quantitativo, } \\
\text { correlacional } \\
\text { e transversal }\end{array}$ & $\begin{array}{l}\text { Investigar se há } \\
\text { relação entre EID’s } \\
\text { e empatia }\end{array}$ & YSQ-S2 & $\begin{array}{l}60 \text { estudantes } \\
\text { universitários }\end{array}$ & $\begin{array}{l}\text { Dentro das quatro dimensoes } \\
\text { avaliadas na empatia foram } \\
\text { identificadas correlações } \\
\text { positivas entre os esquemas de } \\
\text { abandono/instabilidade, } \\
\text { vulnerabilidade ao } \\
\text { dano/doença e autossacrifício, } \\
\text { com a consideração empática } \\
\text { e a angústia pessoal; e dos } \\
\text { equemas de fracasso e inibição } \\
\text { emocional com a angústia } \\
\text { pessoal. Além disso, } \\
\text { correlações ambivalentes } \\
\text { foram identificadas com os } \\
\text { esquemas de } \\
\text { desconfiança/abuso, } \\
\text { isolamento social/alienação, } \\
\text { emaranhamento, } \\
\text { grandiosidade/arrogo } \\
\text { autocontrole/autodisicplina } \\
\text { insuficientes apresentando } \\
\text { correlação positiva com a } \\
\text { angústia pessoal, mas negativa } \\
\text { com a tomada de perspectiva. }\end{array}$ \\
\hline $\begin{array}{l}\text { Borges, } \\
\text { Lopes e } \\
\text { Lopes } \\
(2018)\end{array}$ & $\begin{array}{l}\text { Quantitativo } \\
\text { de caráter } \\
\text { descritivo e } \\
\text { exploratório }\end{array}$ & $\begin{array}{l}\text { Aprofundar os } \\
\text { conhecimentos } \\
\text { sobre o fenômenos } \\
\text { bullying } \\
\text { unversitários e a sua } \\
\text { relação com os } \\
\text { EID's }\end{array}$ & YSQ-S2 & $\begin{array}{c}236 \\
\text { universitários }\end{array}$ & $\begin{array}{l}\text { O } 1^{\circ} \text { domínio esquemático } \\
\text { (desconexão e rejeição) foi } \\
\text { encontrado expressivamente } \\
\text { na amostra, especialmente os } \\
\text { EID’s de desconfiança/abuso, } \\
\text { privação emocional e } \\
\text { isolamento social/alienação. O } \\
2^{\circ} \text { domínio (autonomia e } \\
\text { desempenho prejudicados) } \\
\text { aparece em parte da amostra e } \\
\text { o } 3^{\circ} \text { domínio (limites } \\
\text { prejudicados) de forma menos } \\
\text { expressiva e mais presente no }\end{array}$ \\
\hline
\end{tabular}


Camargos,

Montagnero, Quantitativo

Lopes e

Lima (2020)
Avaliar os EID's predominantes em pacientes bariátricos curso de educação física. O único esquema encontrado do $4^{\circ}$ domínio (orientação para o outro) foi o de autossacrifício, presente apenas no curso de nutrição $\mathrm{O} \quad 5^{\circ}$ domínio (supervigilância e inibição) apareceu em quase todos os grupos de estudantes, especialmente os esquemas de padrões

inflexíveis/hipercriticidade e inibição emocional.

O EID que obteve a maior média geral, e por grupo, foi o de autossacríficio, seguido do esquema de padrões inflexíveis/postura crítica exagerada. $O$ EID que apresentou menor média geral 30 foi $\quad 0 \quad$ de participantes defectividade/vergonha

(20 pré- seguido pelo esquema de bariátricos e fracasso, apesar de no grupo 10 pós- pré-bariátrico a menor média bariátricos) ser do esquema de privação emocional. Os EID's de privação emocional e o de desconfiança/abuso apresentaram valores mais altos entre os participantes pós-bariátricos se comparados com os pré-bariátricos.

A falta de segurança e estabilidade, geralmente existentes em sujeitos que desenvolveram EID's do primeiro domínio (desconexão
Pressi e

Falcke

(2018)
Haack,
Quantitaivo, correlacional e explicativo
O poder preditivo de experiências na família de origem, EID's, amor, ajustamento conjugal e clima familiar para a ocorrência de violência física conjugal
Pesquisa de

Lima e

Ferreira

(2015) levantamento de caráter quantitativo e qualitativo
Avaliar a presença de EID's em usuários de álcool e outras drogas
YSQ-S2 YSQ-S3 372 sujeitos e rejeição) pode ser um preditor que irá infuenciar relações futuras, havendo correlação entre esquemas e violência conjugal.

A prevalência dos esquemas identificados foram os de abandono/instabilidade, defectividade/vergonha, privação emocional, desconfiança/abuso, vulnerabilidade ao dano/doença, emaranhamento,
Quatro usuários de um Centro de Atenção Psicossocial (CAPS) subjugação, autossacrifício, inibição emocional e padrões inflexíveis/hipercriticidade.

Em três participantes também foram encontrados escores significativos no esquema autocontrole/autodisciplina insuficientes. 


$\begin{array}{cclcc}\text { Luz, Santos, } & \text { Quantitativo } & \text { Identificar } & & \text { 236 pessoas } \\ \text { Cazassa e } & \text { e } & \text { diferenças nos } & \text { YSQ-S2 } & \begin{array}{c}\text { (114 do sexo } \\ \text { masculino e }\end{array} \\ \text { Oliveira } & \text { comparativo } & \text { EID's entre homens } & & 123 \text { do sexo } \\ (2012) & \text { transversal } & \text { e mulheres } & & \text { femino })\end{array}$

As mulheres tiveram maior pontuação nos esquemas de fracasso, vulnerabilidade ao dano/doença, autossacrifício, assim como no $2^{\circ}$ (autonomia e desempenho prejudicados) e

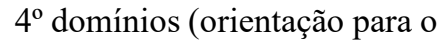
outro). Os homens tiveram maior pontuação no esquema de inibição emocional. Não hove diferença significativa nos outros esquemas.

\begin{tabular}{|c|c|c|c|c|}
\hline $\begin{array}{c}\text { Méa, } \\
\text { Zancanella, } \\
\text { Ferreira e } \\
\text { Wagner } \\
(2015)\end{array}$ & $\begin{array}{l}\text { Quantitativo } \\
\text { com } \\
\text { delineamento } \\
\text { exploratório } \\
\text { e } \\
\text { correlacional }\end{array}$ & $\begin{array}{l}\text { Identificar EID's } \\
\text { presentes em } \\
\text { pacientes internados } \\
\text { devido a tentativa de } \\
\text { suicídio }\end{array}$ & YSQ-S2 & $\begin{array}{l}15 \text { pacientes } \\
\text { comparados } \\
\text { com os } \\
\text { resultados de } \\
25 \text { pessoas de } \\
\text { uma amostra } \\
\text { não clínica }\end{array}$ \\
\hline
\end{tabular}

Paim, Estudo correlação entre Madalena e quantitativo conjugal, assim Falcke com como a correlação (2012) delineamento entre os EID's e as correlacional habilidades de negociação
O grupo clínico apresentou prejuízos em todos os EID's em comparação com o grupo não clínico, estando entre os esquemas com maior escore os EID's de defectividade/vergonha e isolamento social/alienação. Os indíces de violência conjugal cometida pelo sujeito tiveram correlação positiva e significativa com os esquemas de desconfiança/abuso, isolamento social, defectividade/vergonha, dependência/incompetência, emaranhamento, autossacrifício, grandiosidade/arrogo e autocontrole/autodisciplina insuficientes. Os EID's de desconfiança/abuso, emaranhamento, autossacrifício, inflexíveis/hipercriticidade,
163 pessoas casadas ou em YSQ-S3 união estável entre um e 47 anos inflexíveis/hipercriticidade, grandiosidade/arrogo, autocontrole/autodisciplina insuficientes e postura punitiva apresentaram correlação positiva e significativa com os índices de violência conjugal cometida pelo companheiro, ou seja, os esquemas da vítima. No que se refere a negociação, os esquemas de privação emocional, defectividade/vergonha, fracasso, emaranhamento, subjugação autocontrole/autodisciplina insuficientes do sujeito tiveram correlação negativa e significativa com a habilidade de negociação do sujeito e do companheiro. O esquema de dependência/incompetência se 


\begin{tabular}{|c|c|c|c|c|c|}
\hline & & & & & $\begin{array}{l}\text { correlacionou negativamente } \\
\text { somente com a habilidade de } \\
\text { negociação do sujeito, e o } \\
\text { esquema de autossacrifício, } \\
\text { com a habilidade de } \\
\text { negociação do companheiro. }\end{array}$ \\
\hline $\begin{array}{l}\text { Perin e } \\
\text { Ferreira } \\
(2016)\end{array}$ & Quantitativo & $\begin{array}{l}\text { Levantar a } \\
\text { prevalência de } \\
\text { sintomas corporais, } \\
\text { sintomas } \\
\text { depressivos, } \\
\text { sintomas de } \\
\text { ansiedade e EID'S }\end{array}$ & YSQ-S2 & $\begin{array}{c}55 \\
\text { universitários }\end{array}$ & $\begin{array}{l}\text { Com exceção dos EID’s de } \\
\text { inibição emocional, } \\
\text { autocontrole/autodisciplina } \\
\text { insuficientes, } \\
\text { dependência/incompetência, } \\
\text { grandiosidade/arrogo } \\
\text { desconexão, os universitários } \\
\text { que apresentaram no } \\
\text { questionário de esquemas } \\
\text { corporais (BSQ) resultado } \\
\text { normal/leve } \\
\text { moderado/grave, } \\
\text { apresentaram } \\
\text { significante nos diferença } \\
\text { esquemas. }\end{array}$ \\
\hline $\begin{array}{l}\text { Ribas et al. } \\
\text { (2018) }\end{array}$ & Quantitativo & $\begin{array}{l}\text { Avaliar os EID's em } \\
\text { pacientes } \\
\text { enxaqueca }\end{array}$ & YSQ-S3 & $\begin{array}{c}65 \text { pessoas (38 } \\
\text { com } \\
\text { enxaqueca e } \\
27 \text { sem } \\
\text { enxaqueca) }\end{array}$ & $\begin{array}{l}\text { Houve associação } \\
\text { significativa entre enxaqueca e } \\
\text { gênero feminino, bem como, } \\
\text { entre o } 5^{\circ} \text { domínio } \\
\text { (superviligância e inibição), e } \\
\text { os esquemas de padrões } \\
\text { inflexíveis/hipercriticidade e } \\
\text { postura punitiva, em pacientes } \\
\text { com enxaqueca de ambos os } \\
\text { genêros. }\end{array}$ \\
\hline $\begin{array}{l}\text { Rocha, } \\
\text { Formiga e } \\
\text { Lopes } \\
(2014)\end{array}$ & Quantitativo & $\begin{array}{lr}\text { Avaliar } & \text { a } \\
\text { identificação } & \text { de } \\
\text { esquemas } & \text { de } \\
\text { personalidade } & \text { mais } \\
\text { comuns para } & \text { tipos } \\
\text { de crimes } & \text { em } \\
\text { presidiários } & \end{array}$ & YSQ-S2 & $\begin{array}{c}244 \\
\text { presidiários }\end{array}$ & $\begin{array}{l}\text { O esquema que apresentou } \\
\text { maior escore geral foi o de } \\
\text { autossacrifício e a menor } \\
\text { média geral o esquema de } \\
\text { defectividade/vergonha. Nos } \\
\text { subgrupos, conforme crime } \\
\text { pelo qual se encontravam } \\
\text { condenados, os resultados } \\
\text { foram: furto: maior média no } \\
\text { esquema de autossacrifício, } \\
\text { seguido pelo do } \\
\text { abandono/instabilidade } \\
\text { grandiosidade/arrogo, sendo o } \\
\text { menor escore o de } \\
\text { defectividade/vergonha; } \\
\text { roubo: maior média no } \\
\text { esquema de autossacrifício, } \\
\text { seguido por padrões } \\
\text { inflexíveis/hipercriticidade e } \\
\text { abandono/instabilidade, sendo } \\
\text { o menor escore o de } \\
\text { defectividade/vergonha; } \\
\text { homicídio: maior média no } \\
\text { esquema de autossacrifício, } \\
\text { seguido de padrões } \\
\text { inflexíveis/hipercriticidade e } \\
\text { desconfiança/abuso, sendo o } \\
\text { menor escore o de } \\
\text { defectividade/vergonha; }\end{array}$ \\
\hline
\end{tabular}




$\begin{array}{ccl} & & \begin{array}{l}\text { Identificar sintomas } \\ \text { de ansiedade, } \\ \text { depressão e os EIDs }\end{array} \\ \text { Schmidt, } & \text { Quantitativo } & \begin{array}{l}\text { numa amostra } \\ \text { de pacientes }\end{array} \\ \text { Ferreira e } & \text { e } & \text { odontológicos com } \\ \text { Wagner } & \text { comparativo } & \begin{array}{l}\text { sintomas de } \\ \text { disfunção } \\ \text { temporomandibular } \\ \end{array} \\ & & \text { (DTM) }\end{array}$

Identificar sintomas

de ansiedade, numa amostra

Ferreira e

Wagner

(2015) estupro: maior média no esquema de autossacrifício, seguido de abandono/instabilidade e padrões

inflexíveis/hipercriticidade, sendo o menor escore o de dependencia/incompentência; tráfico de drogas: maior média no esquema de autossacrifício, seguido abandono/instabilidade e padões inflexíveis, sendo o menor escore o de fracasso.

Os EID's que apresentaram diferenças entre os grupos foram desconfiança/abuso, subjugação, inibição emocional, bem como privação emocional, isolamento social/alientação e emaranhamento/self odontológicos

(20 com

e 20 sem subdesenvolvido. Três deles

DTM) são do $1^{\circ}$ domínio, sugerindo experiências emocionais negativas na infância de pacientes com DTM.

\begin{tabular}{|c|c|c|c|c|c|}
\hline $\begin{array}{l}\text { Schmidt e } \\
\text { Méa (2017) }\end{array}$ & $\begin{array}{l}\text { Estudo de } \\
\quad \text { caso }\end{array}$ & 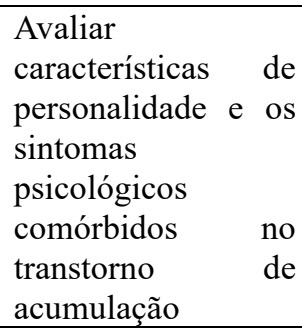 & $\begin{array}{l}\text { YSQ-S2, } \\
\text { inventário } \\
\text { dos estilos } \\
\text { parentais, } \\
\text { entrevista } \\
\text { semi- } \\
\text { estruturada }\end{array}$ & $\begin{array}{c}\text { Paciente com } \\
\text { diagnóstico de } \\
\text { transtorno de } \\
\text { acumulação }\end{array}$ & $\begin{array}{l}\text { Os esquemas com escore } \\
\text { considerados } \\
\text { foram os de prifnificativos } \\
\text { emocional, } \\
\begin{array}{l}\text { social/alienação, } \\
\text { autossacrifício e }\end{array} \\
\begin{array}{l}\text { emocional. inibição } \\
\text { emonal. }\end{array}\end{array}$ \\
\hline $\begin{array}{l}\text { Squefi e } \\
\text { Andretta } \\
(2016)\end{array}$ & $\begin{array}{l}\text { Quantitativo, } \\
\text { transversal e } \\
\text { comparativo }\end{array}$ & $\begin{array}{l}\text { Identificar } \\
\text { diferenças nos } \\
\text { EID's e habilidades } \\
\text { sociais educativas } \\
\text { entre pais e mães }\end{array}$ & YSQ-S3 & $\begin{array}{l}154 \text { pessoas } \\
\text { (46 pais e } 108 \\
\text { mães) com ao } \\
\text { menos um(a) } \\
\text { filho com } \\
\text { idade entre } \\
\text { sete e } 11 \text { anos }\end{array}$ & $\begin{array}{l}\text { As mães demonstraram } \\
\text { escores mais altos que os pais } \\
\text { nos EID's de } \\
\text { dependência/incompetência, } \\
\text { emaranhamento, fracasso e } \\
\text { autossacríficio, sendo três } \\
\text { deles do } 2^{\circ} \text { domínio } \\
\text { (autonomia e desempenho } \\
\text { prejudicados). }\end{array}$ \\
\hline $\begin{array}{l}\text { Vargas e } \\
\text { Pureza } \\
(2019)\end{array}$ & $\begin{array}{l}\text { Quantitativo, } \\
\text { descritivo, } \\
\text { correlacional } \\
\text { e transversal }\end{array}$ & $\begin{array}{l}\text { Avaliar a relação } \\
\text { entre os EID's e o } \\
\text { ajustamento diádico } \\
\text { na conjugalidade }\end{array}$ & YSQ-S3 & $\begin{array}{c}77 \\
\text { participantes } \\
\text { em um } \\
\text { relacionament } \\
\text { o conjugal }\end{array}$ & $\begin{array}{l}\text { Foram observadas as seguintes } \\
\text { correlações entre EID's e } \\
\text { fatores do ajustamento } \\
\text { diádico: os domínios de } \\
\text { desconexão e rejeição, } \\
\text { autonomia e desempenho } \\
\text { prejudicados e direcionamento } \\
\text { para o outro com coesão, } \\
\text { consenso expressão de afeto; o } \\
\text { domínio limites prejudicados } \\
\text { com expressão de afeto e } \\
\text { consenso; e o domínio } \\
\text { supervigilância e inibição com } \\
\text { expressão de afeto, coesão e } \\
\text { satisfação conjugal. }\end{array}$ \\
\hline
\end{tabular}




\section{Discussão}

As datas das publicações avaliadas evidenciam que está ocorrendo o aumento gradativo dos estudos sobre os EID's no Brasil. É possível constatar que a terapia do esquema vem ganhando cada vez mais espaço na literatura brasileira. Um olhar para os EID's e as necessidades emocionais associadas a eles, amplia a compreensão dos transtornos mentais e seus fatores de manutenção (Susin, Carvalho \& Kristensen, 2014).

A variedade das demandas estudadas, conforme apresentado na Tabela 1, demonstra que a terapia do esquema tem sido uma abordagem relevante para o entendimento clínico, o que condiz com a ideia de que o foco de estudo desta linha teórica tenha crescido em diferentes contextos, reforçando o que foi descrito nas revisões realizadas por Masley, Gillanders, Simpson \& Taylor (2012) e Taylor et al. (2016). Além disso, também é possível observar a multidisciplinaridade que a TE tem assumido e a possível relação dos EID's em condições físiológicas, como as apresentadas nessa revisão pelo estudo de Ribas et al. (2018) sobre enxaqueca, o artigo de Schmidt et al. (2015) acerca da disfunção temporomandibular e a pesquisa de Camargos, Montagnero, Lopes e Lima (2020) com pacientes bariátricos.

Cabe destacar que os resultados encontrados, descritos na Tabela 1 , se referem ao grupo investigado e não necessariamente essas informações podem ser generalizadas para todos os indivíduos que fazem parte desses grupos, já que o desenvolvimento dos EID's perpassa questões indivíduais como o temperamento e características familiares (Young et al., 2008). Nesse sentido, é interessante observar que os resultados de determinadas pesquisas corroboram ou contrapõe o entendimento de outros autores. Assim como na pesquisa de Méa, Zancanella, Ferreira e Wagner (2015), a maior severidade nos EID's de pessoas com ideação suicida em relação ao grupo controle também foi observada em pesquisas posteriores, realizadas fora do Brasil (Ahmadpanah et al., 2017; Flink et al., 2017). Segundo esses autores, o nível de sofrimento e dificuldade no manejo das emoções pode ser potencializado pelo conteúdo subjacente dos esquemas, levando à ideação suicida.

Já nos dois artigos analisados que buscaram comparar diferenças nos EID's entre homens e mulheres, foi possível perceber que as mulheres possuem maior escore do que o masculino no $2^{\mathrm{o}}$ domínio esquemático (autonomia e desempenho prejudicados) e também no esquema de autossacríficio (Luz et al., 2012; Squefi \& Andreatta, 2016). Os homens podem apresentar maior escore no esquema de inibição emocional, não havendo diferenças significativas nos outros esquemas (Luz et al., 2012). Além disso, embora o objetivo do estudo de Ribas et al. (2018) não tenha sido a comparação entre os sexos, os autores destacam que o perfil de autoexigência encontrado em sua amostra parece mais presente entre as mulheres, assim como a prevalência de sintomas corporais foi maior no sexo femino na pesquisa de Perin e Ferreira (2016).

Diversas questões culturais e exigências sociais podem estar associadas a esses resultados, de modo que a cultura acaba sendo um aspecto importante nos estilos parentais e atendimento das necessidades emocionais. Portanto, ao compreender os EID's e suas manifestações, é imprescendível levar em conta o contexto em que o indivíduo está inserido (Young et al. 2008). O estudo norte-americano de Shorey, Anderson e Stuart (2012), por exemplo, indicou que 14 esquemas foram mais altos em mulheres e nenhum deles foi significativamente alto no público masculino, o que indica uma diferença dos resultados descritos nos artigos apresentados nessa revisão. A presença mais severa dos esquemas nas mulheres pode estar associada a diferentes quadros de demandas psicólogicas e aos papéis socialmente construídos sobre ser homem e ser mulher (Brenning, Bosmans, Braet \& Thewis, 2012). Essa compreensão não implica apenas nas intervenções com adultos, mas em programas de prevenção com crianças, adolescentes e orientação a cuidadores (Paim \& Rosa, 2016). 
Mesmo grupos da mesma cultura podem apresentar resultados diferentes na predominância de EID's, sofrendo influência de outros aspectos, como por exemplo, a idade. Apesar dos esquemas do $3^{\circ}$ domínio (limites prejudicados) não terem sido expressivos na maior parte da amostra, como os outros domínios foram, na pesquisa selecionada de Borges, Lopes e Lopes (2018), a pesquisa de Mallmann, Lisboa e Calza (2017), realizada com adolescentes, identificou os esquemas de grandiosidade/arrogo

autocontrole/autodisciplina insuficientes, ambos do $3^{\circ}$ domínio, de forma significativa na amostra. Essa diferença pode estar atrelada a diferença na faixa etária entre as pesquisas, visto que algumas características esperadas nesse momento do ciclo vital podem contribuir para a maior prevalência de determinadas respostas nos inventários, tais como a impulsivdade e uma visão mais auto-centrada. Mesmo com essa diferenciação nos resultados, a influência dos EID's no envolvimento com diferentes formas de bullying, tanto como agressor, quanto como vítima é sinalizada pelos artigos.

No que se refere a dependência química, não foi possível identificar EID's prevalentes, visto que a pesquisa de Lima e Ferreira (2015) possuiu uma amostra reduzida de participantes. No entanto, os autores pontuam que os comportamentos aditivos, embora tenham influência multifatorial, podem ser formas que os indivíduos encontram de lidar com os próprios esquemas, já que a existência de EID's possivelmente colabora para a pouca capacidade de manejo das emoções negativas intensas. Essa relação entre a ativação esquemática e o uso de substâncias também foi evidenciada por revisões de literatura, nas quais não foi possível identificar um perfil prevalente de EID's na dependência química nas diferentes pesquisas avaliadas (Cardoso, Antunes \& Cunha, 2019; Maciel, Tractenberg, Habigzang \& Wainer, 2013; Rocha \& Lopes, 2019).

Os relacionamentos afetivos foram trabalhados por quatro artigos, sendo um tema recorrente nessa revisão. De acordo com Young et al. (2008), a manutenção dos esquemas pode ocorrer por meio dessas relações, visto que os padrões familiares - assim como os EID's possuem a tendência de se perpetuar (Young et al., 2008). As pessoas buscam por pessoas que repitam os padrões esquemáticos, podendo sentir um estranhamento com pessoas que não o fazem. Esse processo é conhecido como química esquemática e é um fenômeno importante no atendimento de demandas relacionadas a casais. Geralmente, os relacionamentos possuem um ciclo esquemático, em que a dinâmica da relação reforça os esquemas de cada parceiro (Paim, Madalena, \& Falcke, 2012; Young \& Klosko, 2019).

Os três estudos que abordaram a violência conjugal identificaram que as necessidades emocionais do $1^{\mathrm{o}}$ domínio esquemático (desconexão e rejeição) podem estar associados aos relacionamentos com violência (Barbosa, Côrrea, Zimmer \& Paludo, 2019; Haack et al., 2018; Paim et al. 2012). O EID de defectividade/vergonha, pertencente a esse domínio, obteve uma correlação positiva na amostra de sujeitos que cometeram violência conjugal, enquanto que o EID de desconfiança/abuso surgiu tanto em quem cometeu quanto com quem foi vítima de violência (Paim et al., 2012). A formação de esquemas do $1^{\circ}$ domínio se caracteriza por vivências de vínculos inseguros, com falta de estabilidade emocional e segurança (Young et al., 2008). Pensando na química esquemática e a repetição relacional que ela gera, faz sentido que pessoas em relacionamentos violentos possuam experiências prévias de desamparo e abuso emocional (Paim et al. 2012).

No entanto, o estudo de Paim, Madalena e Falcke (2012) identificou também esquemas pertencentes a outros domínios esquemáticos, como os esquemas de emaranhamento e autossacríficio. Estes foram também citados no estudo teórico de Boscardin e Krintensen (2011) que avaliou duas vinhetas clínicas em situações de amor patológico. Enquanto o emaranhamento se refere a dificuldade de individuação, o autossacríficio denota uma auto responsabilização excessiva pelo outro, 
havendo culpa quando não o faz. Assim, os dois esquemas são caracterizados por uma dificuldade de se posicionar e distanciar de outros indivíduos, podem existir a crença de que o outro não poderá sobreviver sem a sua presença e auxílio (Young \& Klosko, 2019). No entanto, o emaranhamento é pertencente ao $2^{\circ}$ domínio esquemático (autonomia e desempenho prejudicados) estando mais relacionado à falta de independência e autoeficácia, enquanto o autossacríficio, do $4^{\circ}$ domínio esquemático (orientação para o outro), está mais associado à aceitação condicional e busca por validação externa (Young et al., 2008). Já a pesquisa de Barbosa, Côrrea, Zimmer e Paludo (2019), identificou o domínio de supervigilância e inibição como segundo mais pontuado. Os EID's desse domínio estão relacionados com tensão, supressão de emoções e dificuldade de expressar espontaneidade. Muitas vezes são esquemas condicionais que se desenvolveram por conta de outros esquemas nucleares (Young et al., 2008). Pensando na realidade de uma relação com violência, é esperado que temáticas de pessimismo e inflexibilidade estejam mais evidentes na amostra (Cardoso, Bertho \& Paim, 2019).

Publicações recentes acerca da violência conjugal trazem a importância do modelo da terapia do esquema para que se possa intervir de modo efetivo e garantir a segurança dos indivíduos envolvidos nesse tipo de relação (Baldissera, Paim, Predebon \& Feix, 2021). O aumento de estudos sobre a violência conjugal se faz necessário e é estimulado, considerando a frequência com que ocorrem e os impactos diversos que ocasionam (Cardoso et al., 2019). O constante desenvolvimento da TE para casais visa possibilitar que os parceiros possam construir relações em que consigam solicitar, receber e atender as necessidades emocionais um do outro, além de romper o ciclo esquemático em que as situações vividas pelo casal perpetuam constantemente a ativação dos esquemas (Reis \& Andriola, 2019). De acordo com Chan e Tan (2020), isso favorece o apoio mútuo em interesses, valores e sonhos, aumentando a atração e fortalecendo a saúde do casal.

Nesse sentido, um olhar para as necessidades emocionais e a qualidade dos relacionamentos pode ser um fator preventivo no desenvolvimento de EID's, conforme observado no artigo de Berlitz e Pureza (2018) sobre empatia, na pesquisa de Paim et al. (2012) que, além da violência conjugal, investiga habilidades de negociação, e no estudo acerca do ajustamento diádico na conjugalidade de Vargas e Pureza (2019). Essas pesquisas contrapõe as questões de violência abordadas nos outros estudos, trazendo um olhar preventivo para a TE e os relacionamentos interpessoais como um veículo para o suprimento das necessidades emocionais. Isso também é observado no estudo de Paim et al., (2012), que além da violência conjugal investiga as habilidades de negociação dos casais, e na pesquisa de Squefi e Andretta (2016) em que a diferença nos EID's entre pais e mães aborda também as habilidades sociais educativas desses cuidadores. Levar isso em conta é necessário para que programas de prevenção possam ser construídos de modo adequado, promovendo experiências reparadoras para os sujeitos. Educadores, por exemplo, podem assumir um papel importante na construção afetiva que constroem com estudantes (Jager \& Macedo, 2018).

Um ponto importante de diferenciação nos estudos apresentados é o instrumento utilizado para identificação dos EID's, aspecto sinalizado também na revisão de literatura realizada por Taylor et al. (2016) no Reino Unido. As versões reduzidas do questionário de esquemas de Young (Young Schema Questionnaire - YSQ) para adultos contêm, em relação à versão longa, as sentenças mais representativas para o mapeamento de cada esquema e podem ser menos cansativas para o respondente (Paim \& Copetti, 2016). Nos artigos selecionados, dez optaram pelo uso do YSQ-S2 e seis utilizaram o modelo YSQ-S3. Essa diferença pode estar atrelada ao fato de que o YSQ-S3, no Brasil, possui estudos de validação estrutural e adequação semântica mais recentes (Souza, 2019). 
Apenas um dos estudos (Schmidt \& Méa, 2017) fez uso do questionário de estilos parentais. Este visa complementar a avaliação dos EID's por meio da avaliação do relacionamento com os cuidadores e identificação de possíveis padrões (Paim \& Copetti, 2016). A percepção do sujeito sobre seus cuidadores e a maneira com que foram tratados no passado explicita muito de como essa pessoa aprendeu a se relacionar com o mundo e de quais necessidades emocionais podem ter ficado defasadas em seu desenvolvimento (Basso, Fortes, Maia, Steinhorst \& Wainer, 2019). Além disso, o questionário de estilos parentais também permite acessar o grau de sofrimento e entendimento que a pessoa possui na atualidade acerca de experiências passadas. Muitas vezes, o indivíduo se sente mais seguro e confortável para falar sobre os cuidadores do que sobre si mesmo, de modo que temáticas e percepções importantes podem aparecer com esse instrumento (Paim \& Copetti, 2016).

Cabe destacar que existem outros instrumentos que podem ser utilizados na terapia do esquema, como o questionário de esquemas para adolescentes e os inventários de evitação e hipercompensação. $\mathrm{Na}$ prática clínica, estão disponíveis maiores possibilidades de avaliação, como a relação terapêutica e investigação detalhada da história do paciente. A utilização de diferentes recursos pode enriquecer o entendimento individual de cada sujeito (Paim \& Copetti, 2016). Essa avaliação se faz necessária pelo fato de que muitas situações e condições são multifatoriais, não sendo o esquema - por si só - um fator determinante (Méa, Zancanella, Ferreira e Wagner, 2015; Rocha, Formiga e Lopes, 2014).

Nesse sentido, é importante levar em consideração fatores socioculturais que podem gerar influência no contexto investigado, para que se tenha uma compreensão completa dos resultados (Luz et al, 2012; Squefi \& Andretta, 2016). Em alguns casos, por exemplo, as respostas dos questionários podem ser um reflexo do que os indivíduos acreditam ser o socialmente aceito ou esperado, conforme hipotetizado na pesquisa de Rocha, Formiga e
Lopes (2014). Por outro lado, sujeitos que estão em momento de vulnerabilidade emocional, como na pesquisa de Méa et al. (2015), tendem a expressar seus EID's de forma mais genuína (Young et al. 2008). Portanto, as condições de aplicação dos inventários devem ser consideradas ao analisar os resultados (Paim \& Copetti, 2016).

As estratégias de enfrentamento também são fatores que podem interferir nas respostas dos sujeitos. Isso porque, se o indivíduo costuma reagir às ativações emocionais de determinada maneira - como a evitação, por exemplo - essa mesma estratégia pode acontecer no momento da resposta dos inventários, já que eles geralmente mobilizam questões emocionais do respondente (Fassbinder et al., 2016; Paim \& Copetti, 2016; Young et al., 2008). No estudo de Borges et al. (2018) e Rocha et al. (2014), por exemplo, a hipercompensação foi considerada uma possível influência para os resultados obtidos na pesquisa.

Apesar das diferenças entre os estudos, os autores dos artigos selecionados pontuam a importância de pesquisar esquemas iniciais desadaptativos em populações específicas. Esse tipo de trabalho colabora para ampliar o entendimento dos contextos investigados sob o viés da terapia do esquema, possibilitando maior efetividade nas intervenções realizadas e trabalhos de prevenção (Borges, Lopes, \& Lopes, 2018; Lima \& Ferreira, 2015; Luz et al., 2012; Méa et al., 2015; Paim et al., 2012; Perin \& Ferreira, 2016; Rocha et al., 2014; Schmidt \& Méa, 2017; Squefi \& Andretta, 2016). Dessa forma, novos estudos são sugeridos em outros contextos e mesmo nas temáticas abordadas, visto que foram observadas diferentes limitações nas pesquisas apresentadas.

\section{Considerações Finais}

O presente estudo teve como objetivo identificar estudos produzidos no Brasil que tenham investigado esquemas iniciais desadaptativos (EID's) em adultos. Para isso, foi realizada uma revisão narrativa da literatura e apresentados os resultados principais desses 
estudos.

Os artigos avaliados investigaram a existência de EID's e possíveis relações com determinado grupo, nos seguintes contextos: homens e mulheres, pais, mães e habilidades sociais educativas, universitários e empatia, sintomas depressivos, ansiosos e dismorfismo corporal em universitários, bullying em universitários, pacientes com enxaqueca, uso de álcool e outras drogas, transtorno da acumulação, violência conjugal, ajustamento diádico, pacientes bariátricos, pessoas com sintomas de disfunção temporomandibular, pacientes internados por tentativa de suicídio e indivíduos que praticaram crimes. Destaca-se como temática recorrente a violência conjugal, o que é estimulado devido a frequência e gravidade no Brasil. Nos três estudos que abordaram o assunto, foi identificada uma possível associação com $0 \quad 1^{\circ}$ domínio esquemático (desconexão e rejeição).

A diversidade das temáticas encontradas evidencia a variedade de contextos que estão sendo estudados sob a ótica da terapia do esquema. Ao observar as datas de cada publicação, é possível pensar que está acontecendo um aumento gradativo no número de estudos brasileiros com o objetivo de identificar esquemas iniciais desadaptativos em populações específicas. Os autores dos estudos destacam que ampliar esse campo de pesquisa pode favorecer a compreensão do público com o qual se trabalha, possibilitando intervenções terapêuticas e de prevenção mais efetivas. Sendo assim, é indicado que sejam produzidos novos estudos que avaliem EID's em populações específicas.

É necessário também, realizar nova revisão da literatura mais ampla, incluindo também teses, dissertações e publicações internacionais, o que não foi feito nesta pesquisa. A variedade nos métodos utilizados também representou uma limitação para o presente estudo. Não foi possível aprofundar as discussões, associações e hipóteses trazidas nas pesquisas acerca da dinâmica que os esquemas possuem em cada contexto investigado, como por exemplo, o que favorece seu surgimento e quais as suas possíveis consequências. Apesar disso, entende-se que a apresentação dos resultados encontrados pode favorecer uma visão ampla sobre as pesquisas realizadas no Brasil acerca de EID's em demandas e populações específicas, contribuindo para intervenções e novas pesquisas.

\section{Referências}

Ahmadpanah, M., Astinsadaf, S., Akhondi, A., Haghighi, M., Sadeghi Bahmani, D., Nazaribadie, M., ... Brand, S. (2017). Early maladaptive schemas of emotional deprivation, social isolation, shame and abandonment are related to a history of suicide attempts among patients with major depressive disroders. Comprehensive Psychiatry, 77, 71-79. doi: $\underline{\text { 10.1016/j.comppsych.2017.05.008 }}$

Baldissera, D., Paim, K., Predebon, B. M., \& Feix, L. F. (2021). Contribuições da terapia do esquema em relacionamentos conjugais abusivos: uma revisão narrativa. PSI UNISC, 5(1), 51-67. doi: $\underline{10.17058 / \text { psiunisc.v5i1.15386 }}$
Barbosa, T. P., Corrêa, M. A., Zimmer, M., \& dos Santos Paludo, S. (2019). Domínios Esquemáticos Apresentados por Mulheres em Situação de Violência Conjugal. Revista de Psicologia da IMED, 11(2), 51-68. doi: 10.18256/2175-5027.2019.v11i2.2982

Basso, L. A., Fortes, A. B., Maia, C. P., Steinhorst, E., \& Wainer, R. (2019). O efeito dos estilos parentais e esquemas desadaptativos precoces no desenvolvimento da personalidade: uma revisão sistemática. Trends in Psychiatry and Psychotherapy, 41(3), 301-313. doi: 10.1590/2237-6089-2017-0118

Berlitz, D., \& Pureza, J. R. (2018). A relação entre a empatia e os esquemas iniciais desadaptativos. Revista Brasileria de 
Terapias Cognitivas, 14, 31-41. doi: 10.5935/1808-5687.20180005

Bohn, M., Berlitz, D., Oliveira, A. de, \& Pureza, J. da R. (2018). Sofrimento associado ao relacionamento conjugal: olhar da teoria do esquema. Revista Brasileira de Terapias Cognitivas, 14(2), 106-112. doi: $\underline{10.5935 / 1808-}$ 5687.20180015

Boscardin, M. K., \& Kristensen, C. H. (2011). Esquemas desadaptativos em mulheres com amor patológico. Revista de Psicologia da IMED, 3(1), 517-526. doi: 10.18256/21755027/psico-imed.v3n1p517-526

Borges, V.L., Lopes, E.J., \& Lopes, R.F.F. (2018). Relações entre bullying e esquemas iniciais desadaptativos em estudantes universitários. Revista Brasileira de Terapias Cognitivas, 14(1), 57-64. doi: 10.5935/1808-5687.20180008

Brenning, K., Bosmans, G., Braet, C., \& Theuwis, L. (2012). Gender Differences in Cognitive Schema Vulnerability and Depressive Symptoms in Adolescents. Behaviour Change, 29(3), 164-182. doi: 10.1017/bec.2012.15

Camargos, S. P. D. S., Montagnero, A. V., Lopes, R. F. F., \& Lima, A. F. M. (2020). Esquemas iniciais desadaptativos em pacientes pré e pós-bariátricos. Revista Brasileira de Terapias Cognitivas, 16(1), 34-41. doi: 10.5935/1808-5687.20200006

Cazassa, M. J., \& Oliveira, M. S. (2008).

Terapia focada em esquemas: conceituação e pesquisas. Revista de Psiquiatria Clínica, 35, 187-195.

Cazassa, M.J., \& Oliveira, M.S. (2012). Validação brasileira do questionário de esquemas de Young: forma breve. Estudos de Psicologia, 29, 23-31.

Cardoso, B. L. A., Bertho, M. A. C., \& Paim, K. (2019). Até que a morte nos separe: a contribuição da cultura para a manutenção de esquemas iniciais desadaptativos em relacionamentos abusivos. In K. Paim \& B. L. A Cardoso (Orgs.), Terapia do esquema para casais: base teórica e intervenção (pp. 143-163). Porto Alegre: Artmed.

Cardoso, C. O., Antunes, C., \& Cunha, S. C. R. (2019). Esquemas desadaptativos e transtorno por uso de álcool: revisão sistemática. Revista Brasileira de Terapias Cognitivas, 15(1), 19-28. doi: 10.5935/1808-5687.20190005

Carter, J. D., McIntosh, V. V., Jordan, J., Porter, R. J., Frampton, C. M., \& Joyce, P. R. (2013). Psychotherapy for depression: A randomized clinical trial comparing schema therapy and cognitive behavior therapy. Journal of Affective Disorders, 151(2), 500-505. doi: 10.1016/j.jad.2013.06.034

Chan, E. W. L., \& Tan, H. J. R. (2020). Positive psychology couple schema therapy: a new model of couple therapy focusing on reigniting couple attraction via schema therapy and positive psychology. Journal for ReAttach Therapy and Developmental Diversities, 2(2), 61-69, 2020. doi: $10.26407 / 2019$ jrtdd.1.24

Diez, Z. G., Zumalde, E. C., \& Sola, I. O. (2012). Early maladaptative schemas and social anxiety: the moderating effect of avoidant vs. overcompensation coping. [Abrastract]. In Abstracts of the 20th European Congress of Psychiatry, 20th European Congress of Psychiatry (p. 129). Bilbao, Spain.

Falcone, E. M. O. (2014). Terapia do esquema. In W.V Melo (Org.), Estratégias psicoterápicas e a terceira onda em terapia cognitiva (pp. 264-288). Novo Hamburgo, RS: Sinopsys.

Farrell, J. M, Reiss, N., \& Shaw, I. A. (2014). The schema therapy clinican's guide: $a$ complete resource for building and delivering individual, group and integrated schema mode treatment programs. Malden: Wiley-Blackwell.

Fassbinder, E., Schweiger, U., Martius, D., Brand-de Wilde, O., \& Arntz, A. (2016). Emotion Regulation in Schema Therapy and Dialectical Behavior Therapy. Frontiers in Psychology, 7, 1373. doi: $10.3389 /$ fpsyg.2016.01373

Flink, N., Lehto, S. M., Koivumaa-Honkanen, H., Viinamäki, H., Ruusunen, A., Valkonen-Korhonen, M., \& Honkalampi, K. (2017). Early maladaptive schemas and suicidal ideation in depressed patients. The 
European Journal of Psychiatry, 31(3), 87-

92. doi: $10.1016 /$ j.ejpsy.2017.07.001

Haack, K. R., Pressi, J., \& Falcke, D. (2018).

Predictors of Marital Physical Violence:

Personal and Relational

Characteristics. Psico-USF, 23(2), 241-

252. doi: $10.1590 / 1413-82712018230205$

Haan, K. L. B., Fassbinder, E., Hayes, C., \&

Lee, C. W. (2019). A schema therapy

approach to the treatment of posttraumatic

stress disorder. Journal of Psychotherapy

Integration, 29(1), 54-64. doi:

10.1037/int0000120

Halperin, C. F., \& Carneiro, J. C. R. (2016). A teoria do apego e as bases familiares da terapia do esquema. In R. Wainer, K. Paim, R. Erdos, \& R. Andriola (Orgs.), Terapia cognitiva focada em esquemas: integração em psicoterapia (pp. 39-46). Porto Alegre, RS: Artmed.

Hawke, L. D., Provencher, M. D., \& Parikh, S. V. (2013). Schema therapy for bipolar disorder: A conceptual model and future directions. Journal of Affective Disorders, 148(1), 118-122. doi: 10.1016/j.jad.2012.10.034

Hohendorff, J. V. (2014). Como escrever um artigo de revisão de literatura. In S.H.

Koller, M. C. P. P. Couto, \& J. V. Hohendorff (Orgs.), Manual de produção científica (pp. 39-54). São Paulo, SP: Penso Editora Ltda.

Jager, M. E., \& Macedo, J. C. (2018). Relação afetiva professor-aluno e esquemas iniciais desadaptativos em crianças préescolares. Revista Brasileira de Terapias Cognitivas, 14(1). doi: 10.5935/18085687.20180003

Kwak, K.-H., \& Lee, S. J. (2015). A comparative study of early maladaptive schemas in obsessive-compulsive disorder and panic disorder. Psychiatry Research, 230(3), 757-762. doi: 10.1016/j.psychres.2015.11.015

Lima, A. C. R., \& Ferreira, D. V. (2015). Avaliação da prevalência de esquemas iniciais desadaptativos em sujeitos usuários de álcool e outras drogas. Mudanças Psicologia da Saúde, 23, 47-58.
Luz, F. Q., Santos, P. L., Cazassa, M. J., \& Oliveira, M. S. (2012). Diferenças nos esquemas iniciais desadaptativos de homens e mulheres. Revista Brasileira de Terapias Cognitivas, 8(2). doi: 10.5935/1808-5687.20120013

Maciel, L. Z., Tractenberg, S. G., Habigzang, L. F., \& Wainer, R. (2013). Esquemas iniciais desadaptativos no transtorno por uso de álcool. Revista Brasileira de Terapias Cognitivas, 9(2), 101-107. doi: 10.5935/1808-5687.20130014

Mallmann, C. L., Lisboa, C. S. M., \& Calza, T. Z. (2017). Cyberbullying e esquemas iniciais desadaptativos em adolescentes brasileiros. Revista Colombiana de Psicología, 26(2), 313-328. doi: 10.15446/rcp.v26n2.60631

Malogiannis, I. A., Arntz, A., Spyropoulou, A., Tsartsara, E., Aggeli, A., Karveli, S., ... Zervas, I. (2014). Schema therapy for patients with chronic depression: A single case series study. Journal of Behavior Therapy and Experimental Psychiatry, 45(3), 319-329. doi: 10.1016/j.jbtep.2014.02.003

Masley, S. A., Gillanders, D. T., Simpson, S. G., \& Taylor, M. A. (2012). A systematic review of the evidence base for schema therapy. Cognitive Behaviour Therapy, 41(3), 185-202. doi: 10.1080/16506073.2011.614274

Méa, C. P. D., Zancanella, S., Ferreira, V. R. T., \& Wagner, M. F. (2015). Early maladaptive schemas in hospitalized patients for suicide attempt. Revista Brasileira de Terapias Cognitivas, 11(1), 39. doi: 10.5935/1808-5687.20150002

Mendes, M. A., \& Maia, A. M. L. (2019). Teoria do apego e esquemas conjugais. In K. Paim \& B. L. A. Cardoso (Orgs.), Terapia do esquema para casais (pp. 1529). Porto Alegre, RS: Artmed.

Paim, K., \& Copetti, M. E. K. (2016). Estratégias de avaliação e identificação dos esquemas iniciais desadaptativos. In R. Wainer, K. Paim, R. Erdos, \& R. Andriola (Orgs.), Terapia cognitiva focada em esquemas: integração em psicoterapia (pp. 85-127). Porto Alegre, RS: Artmed. 
Paim, K., Madalena, M., \& Falcke, D. (2012). Early maladaptive schemas in marital violence. Revista Brasileira de Terapias Cognitivas, 8(1), 31-39. doi: 10.5935/1808$\underline{5687.20120005}$

Paim, K., \& Rosa, M. (2016). O papel preventivo da terapia do esquema na infância. In R. Wainer, K. Paim, R. Erdos, \& R. Andriola (Orgs.), Terapia cognitiva focada em esquemas: integração em psicoterapia (pp. 169-185). Porto Alegre, RS: Artmed.

Perin, G., \& Ferreira, V. R. T. (2016). Sintomas Depressivos, Sintomas Ansiosos, Dismorfismo Corporal e Esquemas Cognitivos Disfuncionais em Universitários. Revista de Psicologia da IMED, 8(1), 30-37. doi: 10.18256/21755027/psico-imed.v8n1p30-37

Pugh, M. (2015). A narrative review of schemas and schema therapy outcomes in the eating disorders. Clinical Psychology Review, 39, 30-41. doi: 10.1016/j.cpr.2015.04.003

Reis, A. H., \& Andriola, R. (2019). O papel dos esquemas no funcionamento interpessoal. In K. Paim, \& B. L. A. Cardoso (Orgs.), Terapia do esquema para casais: base teórica e intervenção (pp. 314). Porto Alegre, RS: Artmed.

Renner, F., DeRubeis, R., Arntz, A., Peeters, F., Lobbestael, J., \& Huibers, M. J. H. (2018). Exploring mechanisms of change in schema therapy for chronic depression. Journal of Behavior Therapy and Experimental Psychiatry, 58, 97-105. doi: $10.1016 /$ j.jbtep.2017.10.002

Ribas, K. H. S., Ribas, V. R., Barros, S. S. M., Ribas, V. R., Filizola, M. G. N., Ribas, R. M. G., ... Martins, H. A. L. (2018). The participation of Early Maladaptive Schemas (EMSs) in the perception of pain in patients with migraine: A psychological profile. Dementia \& Neuropsychologia, 12(1), 68-74. doi: $\underline{10.1590 / 1980-57642018 \mathrm{dn} 12-010010}$

Rocha, I. C. O., \& Lopes, E. J. (2019). Transtornos por uso de substâncias psicoativas e esquemas iniciais desadaptativos. Revista Brasileira de
Terapia Comportamental E

Cognitiva, 21(1), 76-94. doi: 10.31505/rbtcc.v21i1.1130

Rocha, M. C. O, Formiga, N. S., \& Lopes, E. J. (2014). Esquemas de personalidade mais prevalentes em indivíduos que praticaram crimes. Psic. Rev. São Paulo, 23, 197-217.

Schmidt, D. R., Ferreira, V. R. T., \& Wagner, M. F. (2015). Disfunção

temporomandibular: sintomas de ansiedade, depressão e esquemas iniciais desadaptativos. Temas em Psicologia, 23(4), 973-985. doi: 10.9788/TP2015.4-13

Schmidt, D. R., \& Méa, C. P. D. (2017). Avaliação de sintomas psicológicos no transtorno da acumulação: Um Estudo de Caso. Avaliação Psicológica, 16(3), 268277. doi: 10.15689/ap.2017.1603.12430

Shorey, R. C., Anderson, S. E., \& Stuart, G. L. (2012). Gender differences in early maladaptive schemas in a treatmentseeking sample of alcohol-dependent adults. Substance use \& misuse, 47(1), 108116. doi: 10.3109/10826084.2011.629706

Simpson, S. G., Morrow, E., van Vreeswijk, M., \& Reid, C. (2010). Group Schema Therapy for Eating Disorders: A Pilot Study. Frontiers in Psychology, 1, 182. doi: 10.3389/fpsyg.2010.00182

Souza, L. H. (2019). Adaptação e validação do questionário de esquemas de Youngversão breve (ysq-s3) para uso no Brasil (Dissertação de mestrado). Pontifícia Universidade Católica do Rio Grande do Sul - PUCRS, Porto Alegre, RS, Brasil. Recuperado de https://hdl.handle.net/10923/16521

Squefi, M., \& Andreatta, I. (2016). Esquemas iniciais desadaptativos e habilidades sociais educativas: pais e mães. Revista Brasileira de Terapias Cognitivas, 12(2), 83-90. doi: 10.5935/1808-5687.20160014

Sundag, J., Ascone, L., de Matos Marques, A., Moritz, S., \& Lincoln, T. M. (2016). Elucidating the role of Early Maladaptive Schemas for psychotic symptomatology. Psychiatry Research, 238, 53-59. doi: 10.1016/j.psychres.2016.02.008 
Susin, N., Carvalho, C. S., \& Kristensen, C. H. (2014). Esquemas desadaptativos e sua relação com o transtorno de estresse póstraumático: uma revisão sistemática. Estudos de Psicologia (Campinas), 31(1), 85-96. doi: 10.1590/0103-166x2014000100009

Taylor, C. D. J., Bee, P., \& Haddock, G. (2016). Does schema therapy change schemas and symptoms? A systematic review across mental health disorders. Psychology and Psychotherapy: Theory, Research and Practice, 90(3), 456479. doi: $10.1111 /$ papt.12112

Thiel, N., Jacob, G. A., Tuschen-Caffier, B., Herbst, N., Külz, A. K., Hertenstein, E., ... Voderholzer, U. (2016). Schema therapy augmented exposure and response prevention in patients with obsessivecompulsive disorder: Feasibility and efficacy of a pilot study. Journal of Behavior Therapy and Experimental Psychiatry, 52, 59-67. doi: 10.1016/j.jbtep.2016.03.006

Vargas, L., \& Pureza, J. da R. (2019). Esquemas Iniciais Desadaptativos e
Ajustamento Diádico na Conjugalidade. Revista Brasileira de Terapias Cognitivas, 15(1), 75-83. doi: 10.5935/1808-5687.20190011

Videler, A. C., van Royen, R. J. J., Legra, M. J. H., \& Ouwens, M. A. (2020). Positive schemas in schema therapy with older adults: clinical implications and research suggestions. Behavioural and Cognitive Psychotherapy, 48(4), 481-491. doi: $10.1017 / \mathrm{s} 1352465820000077$

Wainer, R. (2016). O desenvolvimento da personalidade e suas tarefas evolutivas. In R. Wainer, K. Paim, R. Erdos, \& R. Andriola (Orgs.), Terapia cognitiva focada em esquemas: integração em psicoterapia (pp. 15-26). Porto Alegre, RS: Artmed.

Young, J. E., Klosko, J. S., \& Weishaar, M. E. (2008). Terapia do esquema: guia de técnicas cognitivo-comportamentais inovadoras. Porto Alegre, RS: Artmed.

Young, J. E., \& Klosko, J. S. (2019). Reinvente sua vida. (1a. ed.; R. B. Pazzin, Trad.). Novo Hamburgo, RS: Sinopsys. (Trabalho original publicado em 1993).

\section{Dados sobre os autores:}

- William Macedo Fiuza: Psicólogo. Mestrando em Psicologia pela Universidade de Caxias do Sul. Especialista em Terapia Cognitivo-Comportamental.

- Rossane Frizzo de Godoy: Psicóloga. Especialista em Psicoterapia Humanístico-Existencial: Logoterapia (PUC-RS). Mestre em Ciências do Movimento Humano (UFRGS). Doutora em Ciências Pneumológicas (UFRGS). Professora Adjunta da Universidade de Caxias do Sul Programa de Pós-Graduação em Psicologia-Mestrado Profissional.

Declaração de Direito Autoral

A submissão de originais para este periódico implica na transferência, pelos autores, dos direitos de publicação impressa e digital. Os direitos autorais para os artigos publicados são do autor, com direitos do periódico sobre a primeira publicação. Os autores somente poderão utilizar os mesmos resultados em outras publicações indicando claramente este periódico como o meio da publicação original. Em virtude de sermos um periódico de acesso aberto, permite-se o uso gratuito dos artigos em aplicações educacionais e científicas desde que citada a fonte conforme a licença CC-BY da Creative Commons. 